\title{
ADAPTIVE MULTI-RESOLUTION WINDOWING TECHNIQUE FOR LOCALIZED SPATIO-SPECTRAL ANALYSIS
}

\author{
Zubair Khalid, Rodney A. Kennedy, Salman Durrani and Parastoo Sadeghi \\ Research School of Engineering, The Australian National University, Canberra, ACT 0200, Australia \\ Email: \{zubair.khalid, rodney.kennedy, salman.durrani, parastoo.sadeghi\}@anu.edu.au
}

\begin{abstract}
This paper introduces an adaptive, multi-resolution windowing technique that can be used in conjunction with the spatially localized spherical harmonic transform (SLSHT) to process signals on the 2sphere in the spatio-spectral domain. In contrast with the standard formulation, which uses a fixed window, the new windowing technique is able to respond locally to the signal under analysis, that is, be adaptive, and also is formulated to depend on the spectral degree to give it a multi-resolution character. We further enhance its simultaneous spatial and spectral localization by basing the window on a parametric band-limited Slepian maximum spatial concentration eigenfunction. The criterion for window design is to maximize the energy concentration in each spectral component in the spatiospectral domain. A computationally efficient method is also developed to implement the adaptive window design. An example is also provided to demonstrate the superiority of the new adaptive, multiresolution window technique.
\end{abstract}

Index Terms - 2-sphere; unit sphere; spherical harmonic transform; spatio-spectral domain; spatially localized spherical harmonic transform; fast transforms; adaptive; multi-resolution.

\section{INTRODUCTION}

The development of signal processing techniques for signals defined on the 2-sphere finds many applications in various fields of science and engineering. These applications include gravity of topographic data in geophysics [1], 3D beamforming [2] and wireless channel modeling in communication systems [3].

\subsection{Relation to Prior Work}

In the past decade, many space-scale and space-spectral analysis techniques have been developed to analyze the spatially localized variations in signals defined on the 2 -sphere $[1,4,5]$. For example, wavelets have been used as a tool in signal analysis due to the scale and spatial localization intrinsic to many signals and different attempts have been made to extend Euclidean wavelets to the 2 -sphere [5]. The space-scale analysis of a signal using wavelets is characterized by the scale corresponding to different frequency regions, which naturally allows multi-resolution analysis. However, if we are interested in obtaining the localized contribution of a particular spherical harmonic in the global signal, there is no explicit relation between the scale domain that describes the wavelet transform and the spherical harmonic transform.

An alternative to the space-scale analysis is space-spectral (or spatio-spectral) analysis. For spatio-spectral analysis, spatially localized spherical harmonic transform (SLSHT) has been defined in

This work was supported under the Australian Research Council's Linkage Projects funding scheme (project no. LP100100588).
[6]. The SLSHT is the spherical harmonic transform of a signal windowed with an azimuthally symmetric window. Noting that the window function used for spatial localization ideally should be simultaneously concentrated in both the spatial and the spectral domains, it is shown in [6] that the window function obtained from Slepian concentration problem on the sphere [1] serves as a good choice for the window function as it approaches the lower bound imposed by the concentration uncertainty principle on the sphere [7]. However, the use of the same window function for analyzing different spectral components fixes the resolution of the transform in the spatiospectral domain. Furthermore, since the spatio-spectral representation of a signal depends on the window function, a suitable choice of the window function for the specific signal under consideration has not been investigated before.

\subsection{Contributions}

This paper is the first to reveal how to set up an adaptive multiresolution windowing technique that can be used in SLSHT spatiospectral analysis. The spatial window is chosen as the dominant (largest eigenvalue) band-limited Slepian spatial eigenfunction because we argue that it optimally concentrates energy in the spherical harmonic spectral domain and in the 2-sphere spatial domain. As this window takes a single parameter, which is taken as the band-limit parameter, then it is readily adapted to provide a multiresolution capability where the window scale can be tuned to the spectral degree and to localized signal-dependent features. We also present a matrix formulation of the transform technique and provide inversion results that map from the spatio-spectral domain back to the original signal on the 2 -sphere. The contributions conclude with the development of computationally efficient methods and a toy example illustrating the benefits of the new scheme over standard fixed window techniques.

\section{PRELIMINARIES}

\subsection{Signals on the 2-Sphere}

The square integrable complex-valued functions $f(\widehat{\boldsymbol{x}})$ defined on 2-sphere $\mathbb{S}^{2} \triangleq\left\{\boldsymbol{u} \in \mathbb{R}^{3}:\|\mathbf{u}\|=1\right\}$, where $\widehat{\boldsymbol{x}} \equiv \widehat{\boldsymbol{x}}(\theta, \phi) \triangleq$ $(\sin \theta \cos \phi, \sin \theta \sin \phi, \cos \theta)^{T} \in \mathbb{R}^{3}$ is a unit vector which parameterizes a point on the 2-sphere with $\theta \in[0, \pi]$ and $\phi \in[0,2 \pi)$ denoting the co-latitude (or elevation) and longitude (or azimuth) respectively. Here $(\cdot)^{T}$ denotes the vector transpose operation.

The space of square integrable complex valued functions on the sphere forms a complete Hilbert space $L^{2}\left(\mathbb{S}^{2}\right)$ with the inner product $\langle f, h\rangle \triangleq \int_{\mathbb{S}^{2}} f(\widehat{\boldsymbol{x}}) \overline{h(\widehat{\boldsymbol{x}})} d s(\widehat{\boldsymbol{x}})$, where $d s(\widehat{\boldsymbol{x}})=\sin \theta d \theta d \phi$ is the area element, $\overline{(\cdot)}$ denotes complex conjugate and the integration is carried out over $\mathbb{S}^{2}$. The inner product induces a norm $\|f\| \triangleq\langle f, f\rangle^{1 / 2}$ and the functions with finite induced norm are referred to as the signals on the 2 -sphere. 


\subsection{Spherical Harmonics}

The basis for the spectral representation of a signal on the 2-sphere are the spherical harmonics, which are characterized as follows. The Hilbert space $L^{2}\left(\mathbb{S}^{2}\right)$ is separable and spherical harmonics $Y_{\ell}^{m}(\widehat{\boldsymbol{x}})=Y_{\ell}^{m}(\theta, \phi)$ [8], defined for integer degree $\ell \geq 0$ and integer order $m \in[-\ell, \ell]$, form archetype complete orthonormal set of basis functions. We can expand any signal $f \in L^{2}\left(\mathbb{S}^{2}\right)$ as

$$
f(\widehat{\boldsymbol{x}})=\sum_{\ell, m}(f)_{\ell}^{m} Y_{\ell}^{m}(\widehat{\boldsymbol{x}}), \quad \text { where }(f)_{\ell}^{m} \triangleq\left\langle f, Y_{\ell}^{m}\right\rangle
$$

and we have used the shorthand notation $\sum_{\ell, m} \triangleq \sum_{\ell=0}^{\infty} \sum_{m=-\ell}^{\ell}$. The term $(f)_{\ell}^{m}$ is called the spherical harmonic Fourier coefficient of degree $\ell$ and order $m$. For notational simplification, define the infinite dimensional column vector

$$
\mathbf{f}=\left((f)_{0}^{0},(f)_{-1}^{1},(f)_{1}^{0},(f)_{1}^{1},(f)_{2}^{-2}, \ldots\right)^{T}
$$

as the spectral response of the signal containing all spherical harmonic coefficients. The signal $f$ is said to be band-limited at degree $L_{f}$ if $(f)_{\ell}^{m}=0, \forall \ell>L_{f}$. For such a band-limited signal, the spectral response $\mathbf{f}$ is a $\left(L_{f}+1\right)^{2}$ dimensional vector. For an azimuthally symmetric function, such that $f(\theta, \phi)=f(\theta)$, only the zero-order spherical harmonic coefficients of $f$ are non-zero, that is $(f)_{\ell}^{m}=0$ for all $m \neq 0$.

\subsection{Rotations on the 2 -sphere}

Define the rotation operator $\mathcal{D}(\widehat{\boldsymbol{y}})$ with $\widehat{\boldsymbol{y}}=\widehat{\boldsymbol{y}}(\vartheta, \varphi)$ that rotates the azimuthally symmetric function $h(\widehat{\boldsymbol{x}})$ by $\vartheta \in[0, \pi]$ about the $y$-axis followed by $\varphi \in[0,2 \pi)$ about the $z$-axis. Under this rotation operation $\mathcal{D}(\widehat{\boldsymbol{y}})$, the spherical harmonic coefficients of the rotated signal are related to those of the original signal through [8]

$$
(\mathcal{D}(\widehat{\boldsymbol{y}}) h)_{\ell}^{m}=\sqrt{\frac{4 \pi}{2 \ell+1}} \overline{Y_{\ell}^{m}(\vartheta, \varphi)}(h)_{\ell}^{0}, \text { where } \widehat{\boldsymbol{y}}=\widehat{\boldsymbol{y}}(\vartheta, \varphi) .
$$

\subsection{Spatially Localized Spherical Harmonics Transform}

The spatially localized spherical harmonics transform (SLSHT) has been defined as a windowed spherical harmonics transform in [6] to represent the signal jointly in the spatio-spectral domain. SLSHT of degree $\ell$ and order $m$ is defined as the spherical harmonics transform of a signal $f(\widehat{\boldsymbol{x}})$ localized with a rotated azimuthally symmetric function $h(\widehat{\boldsymbol{x}})$ band-limited at $L_{h}$ centered at $\widehat{\boldsymbol{y}}$, i.e.,

$$
g\left(\widehat{\boldsymbol{y}} ; \ell, m ; L_{h}\right) \triangleq \int_{\mathbb{S}^{2}} f(\widehat{\boldsymbol{x}})(\mathcal{D}(\widehat{\boldsymbol{y}}) h)(\widehat{\boldsymbol{x}}) \overline{Y_{\ell}^{m}(\widehat{\boldsymbol{x}})} d s(\widehat{\boldsymbol{x}}) .
$$

The SLSHT $g\left(\widehat{\boldsymbol{y}} ; \ell, m ; L_{h}\right)$ represents the signal jointly in the spatio-spectral domain, which provides information about the contribution of the spherical harmonics in the signal $f(\widehat{\boldsymbol{x}})$ spatially localized within the windowed region. It also reveals the spatially varying contribution of each spectral component to the global spherical harmonic coefficient.

\section{ADAPTIVE MULTI-RESOLUTION WINDOW SLSHT}

\subsection{Slepian Eigenfunction Window}

The formulation of SLSHT $g\left(\widehat{\boldsymbol{y}} ; \ell, m ; L_{h}\right)$ in (3) can be interpreted as the spherical harmonic transform of the spatially localized signal, where the window function $h(\widehat{\boldsymbol{x}})$ provides localization at position $\widehat{\boldsymbol{y}} \in \mathbb{S}^{2}$ through $(\mathcal{D}(\widehat{\boldsymbol{y}}) h)(\widehat{\boldsymbol{x}})$. Therefore, the SLSHT is dependent on the chosen window function $h(\widehat{\boldsymbol{x}})$ and in the conventional setting, (3), this window is fixed (independent of the spatio-spectral coordinates, $\widehat{\boldsymbol{y}}$ and $\ell, m$ and independent of the signal $f(\widehat{\boldsymbol{x}})$ ).
The window is localized spectrally to a band-limit at $L_{h}$ but ideally it should also localize spatially such that its energy is maximally concentrated within a polar cap with maximum co-latitude $\theta_{h}$. We shall see that these two parameters, $L_{h}$ and $\theta_{h}$, are complementary and their product is lower bounded by an uncertainty principle, which also implies we cannot have a strict spatial limit if we have a strict band-limit. In our design we shall use bandlimit $L_{h}$ as the parameter and the window is chosen as the dominant band-limited eigenfunction, denoted $h_{L_{h}}(\widehat{\boldsymbol{x}})$, arising from the Slepian spatial concentration problem [1,9]. Such window functions are close in performance to that constrained by uncertainty principle [7]. The spatial parameter $\theta_{h}$ of the eigenfunction window is related to band-limit $L_{h}$ by

$$
\theta_{h}=\frac{2 \pi}{L_{h}+1} .
$$

Furthermore, we impose the condition of unit energy on the window function, that is, $\int_{\mathbb{S}^{2}}\left|h_{L_{h}}(\widehat{\boldsymbol{x}})\right|^{2} d s(\boldsymbol{x})=\sum_{\ell=0}^{L_{h}}\left|\left(h_{L_{h}}\right)_{\ell}^{0}\right|^{2}=1$.

\subsection{Multi-Resolution Capability}

It is clear that the window band-limit $L_{h}$ should be tuned (that is varied) for each degree $\ell$ of interest within the SLSHT (3). Equally clearly it should be chosen independent of the order $m$. The following definition captures this multi-resolution attribute.

Definition 1 (Multi-Resolution SLSHT). For a signal $f \in L^{2}\left(\mathbb{S}^{2}\right)$, define the multi-resolution SLSHT as the SLSHT using a degree dependent band-limited $L_{h}(\ell)$ eigenfunction window $h_{L_{h}(\ell)}$ :

$$
\begin{aligned}
g_{a}(\widehat{\boldsymbol{y}} ; \ell, m) & \triangleq g\left(\widehat{\boldsymbol{y}} ; \ell, m ; L_{h}(\ell)\right) \\
& =\int_{\mathbb{S}^{2}} f(\widehat{\boldsymbol{x}})\left(\mathcal{D}(\widehat{\boldsymbol{y}}) h_{L_{h}(\ell)}\right)(\widehat{\boldsymbol{x}}) \overline{Y_{\ell}^{m}(\widehat{\boldsymbol{x}})} d s(\widehat{\boldsymbol{x}}) .
\end{aligned}
$$

where $h_{L_{h}(\ell)}$ is the band-limited eigenfunction window arising from the Slepian spatial concentration problem.

Later we develop a suitable criterion to determine the band-limit $L_{h}(\ell)$ of the window function for each degree $\ell$. Before then we complete the theory by presenting the multi-resolution SLSHT distribution and its matrix formulation in Section 3.3, and inversion in Section 3.4.

\subsection{Multi-Resolution SLSHT Distribution}

Define the multi-resolution SLSHT distribution $\mathbf{g}_{a}(\widehat{\boldsymbol{y}})$ as the representation of the signal in the spatio-spectral domain in the form of a vector with the same ordering as in $\mathbf{f}$

$$
\mathbf{g}_{a}(\widehat{\boldsymbol{y}})=\left[g_{a}(\widehat{\boldsymbol{y}} ; 0,0), g_{a}(\widehat{\boldsymbol{y}} ; 1,-1), \ldots, g_{a}\left(\widehat{\boldsymbol{y}} ; L_{f}, L_{f}\right)\right]^{T}
$$

with matrix formulation

$$
\mathbf{g}_{a}(\widehat{\boldsymbol{y}})=\boldsymbol{\Psi}(\widehat{\boldsymbol{y}}) \mathbf{f},
$$

where $\boldsymbol{\Psi}(\widehat{\boldsymbol{y}})$ is the matrix of size $\left(L_{f}+1\right)^{2} \times\left(L_{f}+1\right)^{2}$ with the same ordering as in $\mathbf{f}$ for its columns and rows, and its entries are given by $\Psi_{\ell, s}^{m, t}(\widehat{\boldsymbol{y}}) \in L^{2}\left(\mathbb{S}^{2}\right)$ can be expressed as

$$
\Psi_{\ell, s}^{m, t}(\widehat{\boldsymbol{y}})=\sum_{p, q} \sqrt{\frac{4 \pi}{2 p+1}} \overline{Y_{p}^{q}(\widehat{\boldsymbol{y}})}\left(h_{L_{h}(\ell)}\right)_{p}^{0} T(s, t ; p, q ; \ell, m),
$$

where we have used (1) in conjunction with (2) and

$$
T(s, t ; p, q ; \ell, m) \triangleq \int_{\mathbb{S}^{2}} Y_{s}^{t}(\widehat{\boldsymbol{x}}) Y_{p}^{q}(\widehat{\boldsymbol{x}}) \overline{Y_{\ell}^{m}(\widehat{\boldsymbol{x}})} d s(\widehat{\boldsymbol{x}})
$$

denotes the spherical harmonic triple product [6] and can be computed analytically using Wigner- $3 j$ symbols $[8,10]$. 


\subsection{Multi-Resolution SLSHT Inversion}

Similar to [6], we note that the adaptive SLSHT is invertible and the signal spectral coefficient $(f)_{\ell}^{m}$ can be obtained as a spatial marginal of the adaptive SLSHT distribution component $g_{a}(\widehat{\boldsymbol{y}} ; \ell, m)$ as

$$
(f)_{\ell}^{m}=\frac{1}{\sqrt{4 \pi}\left(h_{L_{h}(\ell)}\right)_{0}^{0}} \int_{\mathbb{S}^{2}} g_{a}(\widehat{\boldsymbol{y}} ; \ell, m) d s(\widehat{\boldsymbol{y}}) .
$$

Alternatively, the signal can be recovered (again in spectral form) using the matrix formulation in (6) as

$$
\mathbf{f}=\mathbf{M}^{-1} \int_{\mathbb{S}^{2}} \boldsymbol{\Psi}^{H}(\widehat{\boldsymbol{y}}) \mathbf{g}(\widehat{\boldsymbol{y}}) d s(\widehat{\boldsymbol{y}}),
$$

where $\mathbf{M} \triangleq \int_{\mathbb{S}^{2}} \boldsymbol{\Psi}^{H}(\widehat{\boldsymbol{y}}) \boldsymbol{\Psi}(\widehat{\boldsymbol{y}}) d s(\widehat{\boldsymbol{y}})$ is a diagonal matrix of size $\left(L_{f}+1\right)^{2} \times\left(L_{f}+1\right)^{2}$ with the same ordering as in $\mathbf{f}$ for its columns and rows. Its diagonal entries are of the form

$$
M_{\ell, \ell}^{m, m}=\sum_{s=0}^{L_{f}} \sum_{p=0}^{L_{h}}(2 s+1)\left|\left(h_{L_{h}(\ell)}\right)_{p}^{0}\right|^{2}\left(\begin{array}{ccc}
\ell & p & s \\
0 & 0 & 0
\end{array}\right)^{2},
$$

where the brackets denote the Wigner- $3 j$ symbols [10]. The inversion in (8) is useful in the spatio-spectral filtering [9], where the inversion method outlined in (7) cannot be employed.

\subsection{Adapting the Window to the Signal}

In this subsection, we develop a criterion to determine the bandlimit $L_{h}(\ell)$ of the window function for each degree $\ell$. We choose band-limit $L_{h}(\ell) \in\left[0, L_{\max }\right]$ such that the relative correlation between the localized signal $f(\cdot)\left(\mathcal{D}(\widehat{\boldsymbol{y}}) h_{L_{h}(\ell)}\right)(\cdot)$ and the spherical harmonic $Y_{\ell}^{m}(\cdot)$ is maximized over all spatial positions $\widehat{\boldsymbol{y}} \in \mathbb{S}^{2}$. This relative correlation is quantified by the ratio of energy in the SLSHT distribution component under analysis to the energy in the other distribution components. Since the resolution of the window function $L_{h}(\ell)$ is dependent on the degree $\ell$ only, it must be chosen such that the relative energy in all orders $m \in[-\ell, \ell]$ is maximized. With this consideration we need to match the window to the spherical harmonic degree only. Define the relative energy measure

$$
\mathcal{S}\left(\ell ; L_{h}\right) \triangleq \frac{\sum_{m=-\ell}^{\ell} \mathcal{E}(\ell, m)}{\left(\sum_{\ell^{\prime}=0}^{L_{f}} \sum_{m^{\prime}=-\ell^{\prime}}^{\ell^{\prime}} \mathcal{E}\left(\ell^{\prime}, m^{\prime}\right)\right)-\sum_{m=-\ell}^{\ell} \mathcal{E}(\ell, m)},
$$

where

$$
\mathcal{E}\left(\ell, m ; L_{h}\right) \triangleq\left\|g\left(\cdot ; \ell, m ; L_{h}\right)\right\|^{2}
$$

denotes the energy of the SLSHT distribution component $g(\widehat{\boldsymbol{y}} ; \ell, m)$ obtained using window of band-limit $L_{h}$. Using the relative energy measure $\mathcal{S}\left(\ell ; L_{h}\right)$ in (10), we develop a criterion to determine the resolution (band-limit) $L_{h}(\ell) \in\left[0, L_{\text {max }}\right]$ of the window function for each $\ell$ as

$$
L_{h}(\ell)=\arg \max _{L_{h}} \mathcal{S}\left(\ell ; L_{h}\right)
$$

\subsection{Efficient Computation}

A computationally efficient method to determine the SLSHT distribution has been devised in [11] for a fixed window, that is, for $L_{h}(\ell)=L_{h}$ for all $\ell$. Here we outline the method to compute the adaptive SLSHT distribution $\mathbf{g}_{a}(\widehat{\boldsymbol{y}})$ and show that the computational complexity does not increase with the consideration of different eigenfunction windows for the different spectral degrees. The implementation of the criterion to find the resolution $L_{h}(\ell)$ of the window function for each $\ell$ requires the computation of energy in each SLSHT distribution component, that is, $\mathcal{E}\left(\ell, m ; L_{h}\right)$ in (11) for each degree $\ell$ and order $m$ and for each $L_{h} \in\left[0, L_{\max }\right]$. Using (5) and (1), noting the effect of rotation in (2) and employing the orthonormality of spherical harmonics, we write $\mathcal{E}\left(\ell, m ; L_{h}\right)$ as

$$
\mathcal{E}\left(\ell, m ; L_{h}\right)=\sum_{p=0}^{L_{h}} \frac{4 \pi}{2 p+1}\left|\left(h_{L_{h}(\ell)}\right)_{p}^{0}\right|^{2} F(p, \ell, m)
$$

where

$$
\begin{aligned}
F(p, \ell, m) & \triangleq \sum_{q=-p}^{p}\left|\sum_{s, t}^{L_{f}}(f)_{s}^{t} T(s, t ; p, q ; \ell, m)\right|^{2} \\
& =\sum_{q=-p}^{p}\left|\int_{\mathbb{S}^{2}} f(\widehat{\boldsymbol{x}}) \overline{Y_{\ell}^{m}(\widehat{\boldsymbol{x}})} Y_{p}^{q}(\widehat{\boldsymbol{x}}) d s(\widehat{\boldsymbol{x}})\right|^{2} .
\end{aligned}
$$

We note that the computation of $F(p, \ell, m)$ is independent of the choice of window function and requires the evaluation of integral $\int_{\mathbb{S}^{2}} f(\widehat{\boldsymbol{x}}) \overline{Y_{\ell}^{m}(\widehat{\boldsymbol{x}})} Y_{p}^{q}(\widehat{\boldsymbol{x}}) d s(\widehat{\boldsymbol{x}})$, which is necessarily needed in the computation of SLSHT distribution with a fixed window $\left(L_{h}(\ell)=\right.$ $\left.L_{h}\right)$ [11] and can be exactly computed with the computational complexity $O\left(L_{f}^{3} L_{h}^{2}\right)$ for all $0 \leq \ell \leq L_{f},|m| \leq \ell$ and all $0 \leq p \leq$ $L_{h},|q| \leq p$, where $L_{h} \in\left[0, L_{\max }\right]$. Once we know $F(p, \ell, m)$, $\mathcal{E}\left(\ell, m ; L_{h}\right)$ can be computed in $O\left(L_{h}\right)$ for each $L_{h} \in\left[0, L_{\max }\right]$ and thus the optimal band-limit $L_{h}(\ell)$ of the window can be determined using the criterion (12) in $O\left(L_{h}\left(L_{\max }\right)\right) \equiv O\left(L_{\max }^{2}\right)$, which is less than the complexity to determine $F(p, \ell, m)$ and, therefore, the overall complexity to compute multi-resolution SLSHT distribution does not alter with the variation of the window with degree $\ell$. The only difference is that we need to compute $F(p, \ell, m)$ for $p \leq L_{\max }$ (instead of $p \leq L_{h}$ ) in case of multi-resolution SLSHT distribution and, therefore, the overall complexity to apply the window matching criterion and compute the SLSHT distribution is $O\left(L_{f}^{3} L_{\max }^{2}\right)$.

\section{ILLUSTRATION}

Here, we illustrate through an example that the proposed adaptive SLSHT distribution using different resolution eigenfunction windows tuned to the spectral degree provides more concentration of the spatially localized spectral contents of the signal in the spatiospectral domain as compared to the SLSHT distribution obtained using a fixed window. In our example, we consider the bandlimited azimuthally symmetric signal $f(\widehat{\boldsymbol{x}})$ shown in Fig. 1(a), which is obtained by band-limiting the signal $f_{1}(\widehat{\boldsymbol{x}})$ given by

$$
f_{1}(\widehat{\boldsymbol{x}})=f_{1}(\theta) \triangleq \begin{cases}\sum_{\ell=7}^{9} Y_{\ell}^{0}(\theta, \phi) & \theta \in[\pi / 8,3 \pi / 8] \\ \sum_{\ell=17}^{19} Y_{\ell}^{0}(\theta, \phi) & \theta \in[5 \pi / 8,7 \pi / 8] \\ 0 & \text { otherwise }\end{cases}
$$

at $L_{f}=32$. Since the signal is azimuthally symmetric, only zeroorder spherical harmonic coefficients $(f)_{\ell}^{0}$ can be non-zero, which are shown in Fig. 1(b). We note that the signal has different spectral components localized in different spatial regions. Since we are seeking the contribution of zero-order spherical harmonics in the spatiospectral domain, we obtain the adaptive SLSHT distribution $\mathbf{g}_{a}(\widehat{\boldsymbol{y}})$ using the eigenfunction windows with band-limits $L_{h}(\ell)$.

The $L_{h}(\ell)$ obtained using criterion (12) is shown in Fig. 1(c) and the adaptive, multi-resolution SLSHT distribution $\mathbf{g}_{a}(\widehat{\boldsymbol{y}})$ is shown in Fig. 1(d) as a function of co-latitude $\theta$ and degree $\ell$, where we have also indicated the spatio-spectral region over which the signal is localized. We have also plotted SLSHT distributions, obtained using the windows with fixed band-limit $L_{h}=4$ and $L_{h}=24$, in Fig. 1(e) and (f), respectively. Ideally the SLSHT distribution should be maximally concentrated in the indicated localized spatio-spectral region, which is the case when we obtain adaptive distribution. Using the window with smaller $L_{h}$ produces leakage in the spatial region and 


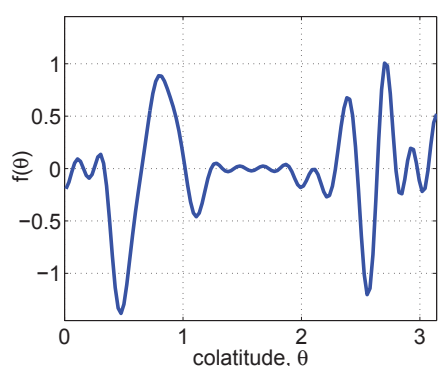

(a)

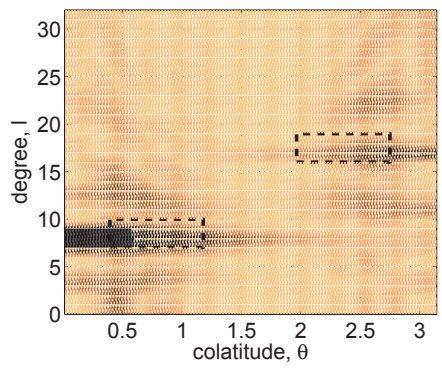

(d)

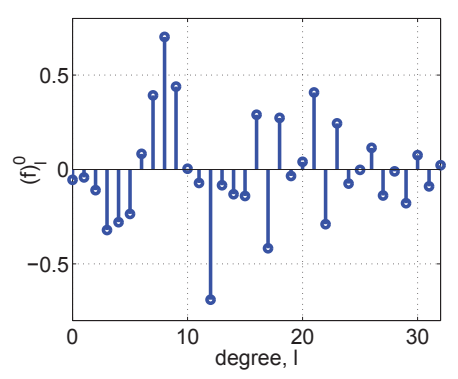

(b)

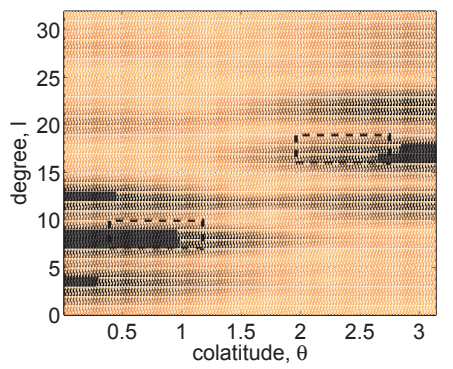

(e)

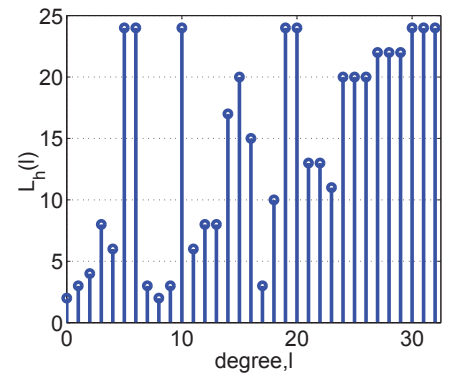

(c)

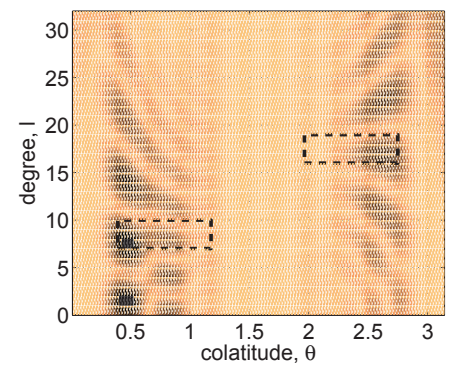

(f)

Fig. 1: (a) Signal $f(\widehat{\boldsymbol{x}})=f(\theta)$ is obtained by band-limiting the signal given in (15) at $L_{f}=32$ with (b) spectral response $(f)_{\ell}^{0}$. (c) The matched resolution (band-limit) $L_{h}(\ell) \in[0,24]$ obtained using the matching criterion in (12), which is then employed to obtain (d) adaptive SLSHT distribution $\mathbf{g}_{a}(\widehat{\boldsymbol{y}})$. The SLSHT distribution obtained using a fixed window is also plotted for (e) $L_{h}=4$ and (f) $L_{h}=24$. The dashed areas denote the regions in spatio-spectral domain over which the signal is localized in spatial and spectral domains.

the window with larger $L_{h}$ causes the energy to be leaked outside the desired spatio-spectral region in the spectral domain. In order to quantify the concentration of the distribution within the desired spatio-spectral region, we also measure the ratio $R$ of energy with in the desired region to the total energy of the distribution. For the SLSHT distribution with fixed window with band-limit $L_{h}=4$ and $L_{h}=24$, we found the ratio to be $R=37.3 \%$ and $R=39.1 \%$, respectively. However, the ratio is $R=54.7 \%$ for adaptive SLSHT distribution, which suggests that the use of multiple windows with resolution matched to the signal under analysis and spectral degree provides more concentration of spatially localized spectral contents in the spatio-spectral domain. The application of proposed technique to real world data presents a work for further research.

\section{CONCLUDING COMMENTS}

The main contributions of the new adaptive multi-resolution SLSHT method has been detailed earlier in Section 1.2, and the effectiveness of the scheme has been demonstrated through the illustrative example. However, extensions of the technique are worth considering. For example, we have argued that the Slepian eigenfunction as the basic window class is a sound choice but whether this proves to be the best choice remains to be determined. Further, the adaptive mechanism, where the window band-limit is adapted to the signal and spectral degree, likewise appears to work well and has manageable complexity but there are many alternatives that could be explored.

\section{REFERENCES}

[1] M. A. Wieczorek and F. J. Simons, "Localized spectral analysis on the sphere," Geophys. J. Int., vol. 162, no. 3, pp. 655-675, May 2005.

[2] D. B. Ward, R. A. Kennedy, and R. C. Williamson, "Theory and design of broadband sensor arrays with frequency invariant far-field beam patterns," J. Acoust. Soc. Am., vol. 97, no. 2, pp. 1023-1034, Feb. 1995.

[3] R. A. Kennedy, P. Sadeghi, T. D. Abhayapala, and H. M. Jones, "Intrinsic limits of dimensionality and richness in random multipath fields," IEEE Trans. Signal Process., vol. 55, no. 6, pp. 2542-2556, Jun. 2007.

[4] M. Simons, S. C. Solomon, and B. H. Hager, "Localization of gravity and topography: constraints on the tectonics and mantle dynamics of Venus," Geophys. J. Int., vol. 131, no. 1, pp. 24-44, Oct. 1997.

[5] Y. Wiaux, L. Jacques, and P. Vandergheynst, "Correspondence principle between spherical and Euclidean wavelets," Astrophys. J., vol. 632, no. 1, pp. 15-28, Oct. 2005.

[6] Z. Khalid, S. Durrani, P. Sadeghi, and R. A. Kennedy, "Spatio-spectral analysis on the sphere using spatially localized spherical harmonics transform," IEEE Trans. Signal Process., vol. 60, no. 3, pp. 1487-1492, Mar. 2012.

[7] F. J. Narcowich and J. D. Ward, "Nonstationary wavelets on the $m$-sphere for scattered data," Appl. Comput. Harmon. Anal., vol. 3, no. 4, pp. 324-336, Oct. 1996.

[8] R. A. Kennedy and P. Sadeghi, Hilbert Space Methods in Signal Processing. Cambridge, UK: Cambridge University Press, Mar. 2013.

[9] Z. Khalid, P. Sadeghi, R. A. Kennedy, and S. Durrani, "Spatially varying spectral filtering of signals on the unit sphere," IEEE Trans. Signal Process., vol. 61, no. 3, pp. 530-544, Feb. 2013.

[10] L. C. Biedenharn and J. D. Louck, Angular Momentum in Quantum Physics: Theory and Application. Reading, MA: Addison Wesley, 1981.

[11] Z. Khalid, R. A. Kennedy, S. Durrani, P. Sadeghi, Y. Wiaux, and J. D. McEwen, "Fast directional spatially localized spherical harmonic transform,” IEEE Trans. Signal Process., vol. 61, no. 9, pp. 2192-2203, May 2013. 